\title{
Efeitos de farelo alimentar fibroso produzido a partir da linhaça em indivíduos com constipação intestinal
}

\author{
Effects of a food produced from the fibrous bran flaxseed in individuals with \\ constipation
}

\author{
Fernanda S. Lopes ${ }^{1}$; Débora S. S. Martins ${ }^{2}$; Jéssica S. Oliveira ${ }^{3}$; Andressa L. Nóbrega ${ }^{4}$; Vicente Maia Pinto Junior ${ }^{4}$
}

\begin{abstract}
Resumo: A preocupação com o bem estar e a melhora da qualidade de vida tem levado as pessoas a consumirem alimentos mais saudáveis os chamados alimentos funcionais, no qual se destaca os que contêm fibra alimentar. Devido às propriedades fibrosas que a linhaça constitui, o presente trabalho teve por objetivo avaliar a eficácia da ingestão de um farelo alimentar rico em fibras, em indivíduos sedentários e não sedentários com constipação intestinal. O presente estudo apresentou caráter laboratorial e experimental com abordagem quantitativa utilizando-se o método da estatística descritiva. $\mathrm{O}$ farelo foi produzido à base da semente de linhaça, com adição de outros ingredientes fibrosos, foi embalado e rotulado, e em seguida distribuído para o consumo. De acordo com os dados obtidos, na análise da composição nutricional do farelo fibroso evidenciou-se um teor apreciável de fibra alimentar. Para a avaliação das características organolépticas do farelo foi observado uma maior aprovação para o sabor, textura e aroma. Quanto à avaliação da função intestinal dos indivíduos após consumir o farelo fibroso foi observado uma melhora significativa em todas as características observadas antes de consumir o farelo, sendo relatado um aumento no número das evacuações. Em relação aos indivíduos sedentários e não sedentários observou-se que independente da prática ou não de atividade física o mesmo foi bastante eficaz em ambos os casos, não sendo relatado nenhum tipo de reação adversa. Diante dos dados concluímos que o farelo fibroso foi bastante eficaz na melhora da constipação intestinal na maioria dos casos estudados.
\end{abstract}

Palavras-chaves: Alimentos saudáveis. Fibra alimentar. Linhaça. Constipação intestinal.

Abstract: The concern with the well-being and improvement of quality of life has led people to consume foods healthier socalled functional foods, which highlights that contain dietary fiber. Due to fibrous properties that flaxseed is, the present study aimed to evaluate the effectiveness of the ingestion of a food rich in fiber, Bran in sedentary individuals and not sedentary with constipation. The present study presented laboratory and experimental character with quantitative approach using the method of descriptive statistics. Bran was produced based on flaxseed, with addition of other fibrous ingredients, was packed and labeled, and then distributed for consumption. According to the data obtained in the analysis of the nutritional composition of the fibrous bran showed an appreciable content of dietary fiber. For the evaluation of the organoleptic characteristics of the bran was observed a greater approval for the taste, texture and aroma. Regarding the assessment of intestinal function of individuals after consuming the fibrous bran was observed significant improvement in all the characteristics observed before consuming the bran, being reported an increase in the number of bowel movements. Compared with sedentary individuals and not sedentary noted that regardless of the practice or not of the same physical activity was quite effective in both cases not reported any type of adverse reaction. In front of the data concluded that the fibrous bran was quite effective in the improvement of constipation in most cases studied.

Keywords: Healthy foods. Dietary fiber. Flaxseed. Constipation.

\footnotetext{
*Autor para correspondência

Recebido para publicação em 20/12/2014; aprovado em 25/01/2015 nandafsl_@hotmail.com.

${ }^{2}$ Graduada em Biomedicina, Faculdades Integradas de Patos-PB. E-mail: suzany_89@ hotmail.com.

${ }^{3}$ Graduada em Enfermagem, Faculdades Integradas de Patos-PB. E-mail: jessica_catole@ hotmail.com.

${ }^{4}$ Graduada em Enfermagem, Faculdades Integradas de Patos, Patos-PB, andressalacerdanobrega@gmail.com

${ }^{5}$ Graduado em Administração de Empresas; E-mail: vicente-maia@ @otmail.com
}

${ }^{1}$ Graduada em Biomedicina das Faculdades Integradas de Patos FIP, Patos, Paraíba, Brasil. Residente: Rua Boileau Dantas Wanderley, 18, Centro, Malta-PB; 


\section{INTRODUÇÃO}

A preocupação com o bem estar e a melhora da qualidade de vida vem levando as pessoas a buscarem uma alimentação mais saudável, o que estimulou beneficamente as indústrias alimentícias a investirem na elaboração de alimentos mais saudáveis e nutritivos, conhecidos popularmente como alimentos funcionais. Os quais são caracterizados como sendo um alimento que em virtude de compostos fisiologicamente ativos, promovem benefícios à saúde, prevenção de doenças e o bem estar físico das pessoas. Sendo considerados indispensáveis ao consumo humano, devendo fazer parte da dieta cotidiana (CARUSO, LAJOLO, MENEZES, 1999; ALMEIDA et al., 2009).

Um dos principais alimentos funcionais consumidos hoje em dia são os que contêm fibras alimentares. Sendo classificados em dois tipos distintos, as fibras solúveis, e as insolúveis características dadas devido as suas propriedades de solubilidade em água. As fibras são definidas como sendo polissacarídeos vegetais da dieta, (celulose, hemicelulose, pectinas, gomas, mucilagens e as ligninas). São encontrados em abundância nos alimentos como grãos, cereais, raízes, folhas e frutos, sendo digerida parcialmente por bactérias que habitam o colón, ou excretadas de forma inalterada nas fezes (FERNANDES et al., 2006).

São considerados alimentos funcionais, pois agem na melhora das atividades vitais e no bom desempenho do trato gastrintestinal. Uma dieta rica em fibras tem o pode de regular o funcionamento do intestino, e evitar a prisão de ventre entre outros agravos como, por exemplo, facilitar o trânsito intestinal (BORGES et a., 2006; ALMEIDA et al., 2009).

A constipação intestinal é um problema que acomete homens, e mulheres em qualquer faixa etária desde crianças até idosos. Ela pode ser um sintoma secundário a alguma patologia como doenças intestinais, ou hipotireoidismo, ou pode ocorrer devido a hábitos alimentares inadequados, sedentarismo ou fatores psicológicos. A não evacuação fecal provoca flatulência, desconforto, distensão abdominal e acúmulo de toxinas no organismo.

Dessa forma, o presente teve como objetivo avaliar a eficácia, e os valores nutricionais de um farelo alimentar fibroso produzido a partir da semente de linhaça, para auxiliar na prevenção e no tratamento de indivíduos com constipação intestinal.

\section{MATERIAL E MÉTODOS}

O presente estudo apresentou caráter laboratorial e experimental com abordagem quantitativa utilizando-se o método da estatística descritiva. $\mathrm{O}$ experimento compreendeu duas fases o que incluiu:

$1^{a}$ fase: Confecção e Análise do composto fibroso, proveniente da linhaça, com suas variações quanto ao teor de fibras e demais nutrientes, está etapa foi realizada na Universidade Federal de Campina Grande - UFCG - campus/ Patos-PB.

$2^{\text {a }}$ fase: Compreendeu a fase experimental da pesquisa, o que incluiu a participação de seres humanos.

A pesquisa foi realizada com uma parte representativa da clientela da Academia FORMA KORPUS, localizada na cidade de MALTA- PB e com indivíduos que não praticavam nenhuma atividade física. A amostra foi composta por 20 pessoas de ambos os gêneros, com faixa etária de 18 á 35 anos, sendo que 10 indivíduos eram clientes da academia e os outros 10 eram sedentários, ambos moradores da cidade de MALTA-PB.

Quanto aos critérios de inclusão, fizeram parte do estudo indivíduos na faixa etária entre 18 a 35 anos, de ambos os sexos, portadores de problemas intestinais diagnosticados como constipação de acordo com os critérios estabelecidos através do questionário previamente aplicado (APÊNDICE C). Quanto aos critérios de exclusão, não participaram da pesquisa indivíduos que não estavam entre a faixa etária estabelecida e que não apresentavam distúrbios intestinais.

A matéria prima utilizada para a produção do farelo fibroso foi composta por: $(55 \%)$ de farinha de linhaça dourada (como principal ingrediente), (25\%) de aveia em flocos grossos e (20\%) de farelo de trigo. As mesmas foram adquiridas no mercado público da cidade de Patos- PB, sendo transportadas para o Laboratório de ciências básicas, das Faculdades Integradas de Patos-PB. No qual foram então processada para a obtenção da amostra fibrosa, para posterior análise da composição nutricional da mesma.

Inicialmente, foi feita a secagem da matéria prima em estufa sob ventilação forçada a $65^{\circ} \mathrm{C}\left(+-2^{\circ} \mathrm{C}\right)$ durante $10 \mathrm{~h}$, sendo posteriormente realizada a moagem em moinho tipo industrial para obtenção das farinhas, em seguida foi realizado sua estocagem em sacos plásticos individuais, posteriormente toda a matéria prima foi unida em um só recipiente, e homogeneizada, e em seguida acondicionada em embalagens individuais de $50 \mathrm{~g}$, rotulada em embalagem personalizada, onde uma parte da amostra foi para análise laboratorial e a outra parte foi entregue para o consumo aos indivíduos que participaram da pesquisa.

A realização da análise da composição nutricional da amostra do farelo foi feita no Laboratório de pesquisa de zootecnia, do Departamento da pós-graduação de zootecnia, pertencente ao Campus Patos-PB, da Universidade Federal de Campina Grande/UFCG. A análise química do composto fibroso constou da composição centesimal, sendo realizadas conforme o método descrito pelo INSTITUTO ADOLFO LUTZ como segue abaixo:

- Umidade: o teor de umidade foi determinado em estufa a $105^{\circ} \mathrm{C}$ até peso constante;

- Cinzas: o teor de cinzas foi determinado em forno mufla à $550^{\circ} \mathrm{C}$, até peso constante;

- Energia: - resultou de determinações calorimétricas diretas, isto é, da determinação do valor calórico do alimento efetuado na bomba calorimétrica;

-Carboidratos: seguiu o método por diferença;

- Lipídios: foi obtido pela extração contínua com solvente orgânico em extrator SOXHLET;

- Proteínas: foi realizada pelo método de KJELDHAL, a determinação foi feita com recurso a uma digestão com ácido num digestor apropriado seguido de uma destilação;

- Fibras: através da determinação do método Fibra alimentar solúvel e insolúvel - Método enzimáticogravimétrico.

Os dados foram analisados de acordo com a literatura pertinente ao tema. A análise foi embasada nas informações contidas no questionário que seguiu a sistematização das respostas encontradas. 
O estudo foi submetido á aprovação do comitê de Ética e Pesquisa (CEP) envolvendo seres humanos das Faculdades Integradas de Patos - FIP. A pesquisa obedeceu aos critérios da Resolução no 196/96 aprovada pelo Conselho Nacional de Saúde - Ministério da Saúde (CNS - MS) (BRASIL, 1996), que trata de pesquisa envolvendo seres humanos e assegura a garantia de privacidade e anonimato ao entrevistado. Garantiu ainda, a liberdade de desistir da pesquisa a qualquer momento e de receber todos os esclarecimentos desejados. Estes direitos foram assegurados aos participantes mediante a assinatura do Termo de Consentimento Livre e Esclarecido (TCLE) em duas vias (APÊNDICE A). Foi entregue também junto ao CEP das FIP e a Secretaria Geral (responsável pela instituição) o termo de compromisso do pesquisador (APÊNDICE B), que declarava a responsabilidade do cumprimento das normas vigentes.

\section{RESULTADOS e DISCUSSÃO}

No presente trabalho foram avaliados 20 indivíduos, diagnosticados constipados, de ambos os gêneros, com faixa etária de 18 a 35 anos, sendo que 10 indivíduos praticavam atividade física e os outros 10 eram sedentários, ambos moradores da cidade de Malta-PB.

A constipação intestinal é considerada como sendo um distúrbio gastrointestinal caracterizado pela diminuição da frequência das evacuações em intervalos de tempo superior às 48hs, o que resulta no aumento da absorção de água pelas paredes do cólon, resultando em fezes endurecidas e de volume pequeno. Devido a sua alta prevalência a mesma está sendo considerada um problema de saúde pública. A preocupação com o bem estar e a melhora da qualidade de vida, tem levado as pessoas a buscarem uma alimentação mais saudável, o que incentivou beneficamente as indústrias alimentícias a investirem na produção de alimentos mais saudáveis, em especial os alimentos funcionais.

Os dados apresentados na tabela 1 expressa às informações referentes a análise da composição nutricional do farelo fibroso produzido, para $100 \mathrm{~g}$ de parte comestível observa-se em sua composição (5\%) de umidade, $(3,22 \%)$ de cinzas, (413) kcal, $(19,19 \mathrm{~g})$ de proteínas, (21g) de lipídeos, $(40 \mathrm{~g})$ de carboidratos e um teor apreciável de $(21,41 \mathrm{~g})$ de fibra alimentar.

Tabela 1. Composição do farelo fibroso por $100 \mathrm{~g}$ de parte comestível.

\begin{tabular}{lcc} 
COMPOSIÇÃO & UNIDADE & RESULTADO \\
\hline Umidade & $\%$ & 5,0 \\
Cinzas & $\%$ & 3,22 \\
Energia & $\mathrm{kcal}$ & 413 \\
Proteínas & $\mathrm{g}$ & 19,19 \\
Lipídeos & $\mathrm{g}$ & 21 \\
Carboidratos & $\mathrm{g}$ & 40 \\
Fibra alimentar & $\mathrm{g}$ & 21,41 \\
\hline
\end{tabular}

De acordo com Dultra (2007) para um alimento ser considerado saudável ele precisa suprir todas as exigências que o corpo necessita, ou seja, têm que conter vários tipos de nutrientes como, energia, proteínas, lipídeos, carboidratos, fibra alimentar entre outros. No farelo fibroso produzido observou-se um teor apreciável de fibra alimentar.
Para Reis (2003) as fibras são indispensáveis ao consumo humano, pois as mesmas são consideradas um dos principais compostos funcionais, atuando no sistema digestivo e na prevenção da constipação intestinal decorrente ao aumento do bolo fecal e do tempo de trânsito intestinal, agindo desta forma no bom desempenho do trato gastrointestinal.

$\mathrm{Na}$ tabela 2, estão expressas as informações referentes à avaliação feita pelos indivíduos com relação às características organolépticas do farelo consumido, observouse que $(95 \%)$ dos indivíduos aprovaram o sabor e apenas $(5 \%)$ não aprovaram, (100\%) gostaram da textura, (100\%) apreciaram o aroma, $(75 \%)$ sentiram facilidade em consumir e $(25 \%)$ apresentaram dificuldade no consumo.

Tabela 2. Avaliação das características organolépticas do farelo fibroso

\begin{tabular}{ccc}
\hline .CARACTERÍSTICAS & $\begin{array}{c}\text { \% DE } \\
\text { APROVAÇÃO }\end{array}$ & $\begin{array}{c}\text { \%DE } \\
\text { REPROVAÇÃo }\end{array}$ \\
\hline Sabor & $95 \%$ & $5 \%$ \\
Textura & $100 \%$ & ---- \\
Aroma & $100 \%$ & ---- \\
Fácil de consumir & $75 \%$ & $25 \%$ \\
\hline
\end{tabular}

Segundo Carrara et al. (2009) os seres humanos se diferem dos demais seres vivos, pois os mesmos ao se alimentarem não buscam apenas suprir as necessidades orgânicas de nutrientes, e sim a de alimentos palpáveis aqueles fáceis de consumir, com sabor, textura e aromas prazerosos ao ingerir. Logo o alimento como fonte de prazer é considerado uma abordagem importantíssima para a promoção da saúde por meio da alimentação.

Nesta pesquisa observou-se uma aceitação apreciável quanto às características organolépticas do farelo, o que contribuiu o consumo pelos indivíduos.

Na tabela 3 pode-se observar a avaliação da função intestinal dos indivíduos antes de consumir o farelo fibroso, no qual todos os indivíduos da pesquisa (100\%) apresentavam trânsito intestinal lento, dor/dificuldade ao evacuar, fezes duras e demoradas e menos de 3 evacuações por semana.

Tabela 3. Avaliação da função intestinal dos indivíduos antes de consumir o farelo fibroso.

\begin{tabular}{lc}
\hline \multicolumn{1}{c}{ DADOS } & \% DE INDIVIDUOS \\
\hline Trânsito intestinal lento & $100 \%$ \\
Dor/dificuldade ao evacuar & $100 \%$ \\
Fezes duras/demorada & $100 \%$ \\
Menos de 3 evacuações por & $100 \%$ \\
semana & \\
\hline
\end{tabular}

Segundo Cota e Miranda (2006), nos últimos anos, devido à sua elevada prevalência, a constipação intestinal crônica (CIC) vem sendo considerada como um problema de saúde pública. Trata-se de uma doença que atinge indivíduos de qualquer idade e apresenta repercussões clínicas que não requerem tratamento de emergência. É responsável por cerca de $3 \%$ das queixas registradas em consultórios de pediatria geral e quase $25 \%$ dos casos de atendimento nos ambulatórios de gastrenterologia pediátrica. Suas causas estão diretamente relacionadas aos hábitos alimentares e ao padrão de vida moderno, que acompanharam a evolução do processo industrial de produção de alimentos, a falta de tempo para 
realizar refeições adequadas e os modismos culturais, contribuintes principais da redução da quantidade de fibras insolúveis dos alimentos.

Para Adibi et al. (2007), mesmo sendo considerada uma doença autolimitada e com resolução na própria infância, a constipação intestinal crônica pode persistir até a idade adulta. A cronicidade dos sintomas, a falta de orientação terapêutica adequada e o uso abusivo de laxantes podem ter como consequências o surgimento de outros problemas como: doença diverticular do cólon, hemorroidas, fissuras anais e fecal mas com impactação fecal.

De acordo com Collete; Araújo; Madruga (2010), as prevalências de constipação intestinal variam, principalmente, de acordo com o local e o critério diagnóstico utilizado. Dados da América do Norte mostraram uma variação entre $2 \%$ e $27 \%$ na prevalência dessa doença e nos países da Europa e Oceania entre 5\% e 35\%. No Brasil, não existem dados publicados de prevalência na população geral, visto que os estudos encontrados na literatura foram todos realizados em subgrupos, como lactentes, adolescentes e mulheres na menopausa.

$\mathrm{Na}$ tabela 4, observamos a avaliação da função intestinal dos indivíduos após consumir o farelo fibroso, no qual a maioria dos indivíduos participantes da pesquisa apresentaram excelentes resultados (90\%) melhoram o trânsito intestinal, $(100 \%)$ tiveram ausência de dor/dificuldade ao evacuar, (100\%) tiveram ausência de fezes duras e demoradas e (90\%) realizaram mais de 3 evacuações por semana.

Tabela 4. Avaliação da função intestinal dos indivíduos após consumir o farelo fibroso.

\begin{tabular}{lc}
\multicolumn{1}{c}{ DADOS } & \% DE EFICÁCIA \\
\hline $\begin{array}{l}\text { Melhora do trânsito intestinal } \\
\begin{array}{l}\text { Ausência de dor/dificuldade ao } \\
\text { evacuar }\end{array}\end{array}$ \\
$\begin{array}{l}\text { Ausência de de } \\
\text { duras/demorada }\end{array}$ & $100 \%$ \\
$\begin{array}{l}\text { Ocorrência de mais de } \\
\text { evacuações por semana }\end{array}$ & $100 \%$ \\
\hline
\end{tabular}

Segundo Lacerda e Pacheco, (2006), a modificação da dieta pode consistir em acrescentar fibras para alcançar uma dieta rica em fibras ( $25 \mathrm{~g}$ de fibras) e suplementos de líquidos (até 1.5-2.0 1/dia), pode melhorar a frequência das fezes e diminuir a necessidade de laxantes. Collete; Araújo; Madruga (2010) ressaltam que nos dias atuais as competências atribuídas ao homem nos seus campos de trabalho, escolar e familiar têm causado interferência em sua qualidade de vida, como por exemplo, falta de horários estabelecidos para as refeições e tempo disponível para a prática de exercícios físicos.

Peppas et al. (2008), afirma que ao mesmo tempo é observada uma mudança nos hábitos alimentares da população, onde se percebe um aumento no consumo de produtos refinados, industrializados com baixo teor de fibras alimentares, contidos em alimentos como cereais integrais, frutas, verduras e hortaliças. Esta mudança tem propiciado o aumento da incidência das "doenças de civilização", sendo a constipação intestinal uma delas.

Carvalho et al. (2006), relatam que a constipação intestinal funcional, está relacionada a fatores como maus hábitos alimentares, sedentarismo e inibição do reflexo de evacuação.

Peppas et al. (2008), descrevem que é recomendada uma ingestão de pelo menos oito copos por dia de líquidos, como água e sucos, por exemplo, pois têm papel no peso e maciez das fezes, além de contribuir para o aumento do número de reflexos gastrológicos e proporcionar uma lubrificação intestinal.

A tabela 5 expressa a avaliação da função intestinal dos indivíduos sedentários e não sedentários em relação à funcionalidade do farelo fibroso, no qual a maioria dos indivíduos participantes da pesquisa apresentaram excelentes resultados $(80 \%)$ dos indivíduos sedentários e (100\%) dos indivíduos não sedentários apresentaram trânsito intestinal rápido, e em relação à sensação de saciedade por longo tempo e alívio dos gases intestinais todos expressaram um valor de $(100 \%)$ quanto à funcionalidade do farelo.

Tabela 5. Comparação entre os indivíduos sedentários e não sedentários em relação a funcionalidade do farelo.

AVALIAÇÃO \% DOS SEDENTÁRIOS QUE \% DOS NÃO SEDENTÁRIOS QUE

APRESENTARAM

Trânsito intestinal rápido

Sensação de saciedade por longo

tempo

Alívio dos gases intestinal

\section{$80 \%$}

$100 \%$

$100 \%$ APRESENTARAM

$100 \%$

$100 \%$
Para Collete; Araújo; Madruga (2010), o sedentarismo e a limitação das atividades físicas decorrentes dos hábitos de vida moderna, como o uso de controle remoto, computadores, vídeo game, televisão, elevador, etc., que contribuem para a diminuição de esforços do ser humano na realização de suas tarefas diárias, também podem ser responsáveis pela diminuição da motilidade intestinal. Abreu et al., (2008) afirmam que a constipação intestinal crônica deve ser considerada como "uma doença da civilização, criada pelo próprio homem, que hoje utiliza drogas como forma de contorná-la".

Rodriguez et al. (2009), comentam que não existem evidências científicas do papel da atividade física para a melhora do quadro de constipação. Mas, conforme descrito por Cota e Miranda (2006), o exercício físico promove motilidade intestinal, mudanças hormonais, melhora do tônus muscular da musculatura pélvica e abdominal, auxiliando a eliminação das fezes após esforço. E, além disso, o gasto energético que se tem com a prática do exercício, faz com que haja um aumento da ingestão de calorias e, por consequência, do consumo de alimentos fontes de fibras.

A tabela 6 relata sobre às possíveis reações adversas ocasionadas pelo consumo do farelo fibroso, no qual se observa que nenhum dos participantes da pesquisa apresentou reações adversas. 
Tabela 6. Reações adversas após o consumo do farelo.

\begin{tabular}{ccc}
\hline REAÇÕES & \% QUE APRESENTARAM & \% QUE NÃO APRESENTARAM \\
\hline Mal estar & ---- & $\mathbf{1 0 0 \%}$ \\
Diarreia & --- & $\mathbf{1 0 0 \%}$ \\
Alergia & --- & $\mathbf{1 0 0 \%}$ \\
Dores abdominais & ---- & $\mathbf{1 0 0 \%}$ \\
\hline
\end{tabular}

Não foi constatado nenhum efeito adverso durante o consumo do farelo. Ao consultar a literatura não foram encontrados dados referentes à ingestão de produtos à base da linhaça, mesmo em doses altas. Há algumas ressalvas quanto o consumo das sementes inteiras pelo conteúdo de algumas proteínas, como lineína, que têm ação antagonista à piridoxina.

\section{CONCLUSÕES}

Diante de todos os dados evidenciou-se que o farelo fibroso produzido a partir da linhaça com adição de outros compostos fibrosos, independente da prática ou não de exercício físico, foi bastante eficaz na melhora da constipação intestinal funcional na maioria dos casos dos indivíduos estudados. Entretanto, conclui-se que o surgimento da constipação intestinal funcional na maioria dos casos está diretamente relacionada à falta de uma alimentação adequada.

\section{REFERÊNCIAS BIBLIOGRÁFICAS}

ABREU, A. S. D; Estudo comparativo do consumo semanal de alimentos fontes de fibras em dois bairros de Porto Velho - RO. Saber Científico, Porto Velho, v. 1, n. 2, p. 189-200, 2008.

ADIBI, P.; BEHZAD, E.; PIRZADEH, S.; MOHSENI M. Bowel habit reference values and abnormalities in young Iranian healthy adults. Dig Dis Sci, n. 52, p. 1810-1813, 2007.

ALMEIDA, C. L.; BOAVENTURA, G. T.; GUZMAN SILVA, M. A; A linhaça (Linumusitatissimum) como fonte de ácido $\alpha$-linolênico na formação da bainha de mielina. Nutrição, .v.22, n.5, set.- out., 2009.

BORGES,V.C. Alimentos funcionais: Prébióticos, Próbióticos, Fitoquimicos e Simbióticos. Em WAITZBERG, D.L, Nutrição oral, enteral e parenteral na prática clinica. $3^{a}$ ed. São Paulo: Ed. Atheneu, p.1495-1509, 2006.

CARRARA, C. L.; ESTEVES A. P.; GOMES, R. T.; GUERRA, L. L. Uso da semente de linhaça como nutracêutco para prevenção e tratamento da arterosclerose. Revista Eletrônica de Farmácia. V.4, p. $1-9,2009$.

CARUSO, L.; LAJOLO, M. F.; MENEZES, E.W. Modelos esquemáticos para avaliação da qualidade analítica dos dados nacionais de fibra alimentar. Cienc. Tecnol. Aliment. v.19, n. 3, 1999.
CARVALHO, E. B.; VITOLO, M. R.; GAMA, C. M.; LOPEZ, F. A.; TADDEI, J. A.; MORAIS, M. B. Fiber intake, constipation, and overweight among adolescents living in Sao Paulo City. Nutrition, v.22, p. 744-749, 2006.

COLlETE, V. L.; ARAÚJO, C. L.; MADRUGA, S. W. Prevalência e fatores associados à constipação intestinal: um estudo de base populacional em Pelotas, Rio Grande do Sul, Brasil, 2007. Caderno de saúde pública, Rio de Janeiro, v. 26, n. 7, p. 1391-1402, jul. 2010.

COTA, R. P.; MIRANDA, L. S. Associação entre constipação intestinal e estilo de vida em estudantes universitários. Revista Brasileira Nutrição Clínica, v. 21, n. 4, p. 296-301, 2006.

DUTRA, E. S. et al. Alimentação Saudável e Sustentável. Modulo11. Brasilia: Universidade de Brasilia,P. 92, 2007.

FERNANDES, L. R.; XISTO, M. D.; PENNA, I. M. M.; LEAL, M. C.; PORTUGAL, L. R.; LEITE, J. I. A. Efeito da goma guar parcialmente hidrolizada no metabolismo de lipídeos e na aterogênese de camundongos. Rev. Nutr. v.19, n.5, 2006.

LACERDA, F. V.; PACHECO, M. T. T. A ação das fibras alimentares na prevenção da constipação intestinal. In: ENCONTRO LATINO AMERICANO DE INICIAÇÃO CIENTÍFICA, 10., 2006, ENCONTRO LATINO AMERICANO DE PÓS-GRADUAÇÃO UNIVERSIDADE DO VALE DO PARAÍBA, 6., 2006, São José dos Campos. Resumos... São José dos Campos: Univap, 2006. p. 2466-2469.

PEPPAS, G.; ALEXIOU, V. G.; MOURTZOUKOU, E.; FALAGAS, M. E. Epidemiology of constipation in Europe and Oceania: a systematic review. BMC Gastroenterol, v. 8, n. 5, 2008.

REIS, N. T. Nutrição Clinica: Sistema Digestório. $3^{a}$ ed. Rio de Janeiro: Rubio. 2003.

RODRIGUEZ, T. N.; DANTAS-JÚNIOR, J. P.; MORAESFILHO, J. P. P. Constipação Intestinal Funcional. Revista Brasileira de Medicina, v. 66, n. 12, p. 77-84, dez. 2009 